\title{
Local Expansion of Donation After Circulatory Death Kidney Transplant Activity Improves Waitlisted Outcomes and Addresses Inequities of Access to Transplantation
}

\author{
B. Mirshekar-Syahkal ${ }^{1}$, D. Summers ${ }^{1}$, \\ L. L. Bradbury ${ }^{2}$, M. Aly ${ }^{1}$, V. Bardsley ${ }^{3}$, M. Berry ${ }^{4}$, \\ J. M. Norris ${ }^{1}$, N. Torpey ${ }^{4}$, M. R. Clatworthy ${ }^{4}$, \\ J. A. Bradley ${ }^{1}$ and G. J. Pettigrew ${ }^{1, *}$ \\ ${ }^{1}$ University Department of Surgery, Addenbrooke's \\ Hospital, Cambridge, UK \\ ${ }^{2} \mathrm{NHS}$ Blood and Transplant, Bristol, UK \\ ${ }^{3}$ Department of Histopathology, Addenbrooke's Hospital, \\ Cambridge, UK \\ ${ }^{4}$ Department of Renal Medicine, Addenbrooke's Hospital, \\ Cambridge, UK \\ ${ }^{*}$ Corresponding author: Gavin J. Pettigrew, \\ gjp25@cam.ac.uk
}

This is an open access article under the terms of the Creative Commons Attribution-NonCommercial-NoDerivs License, which permits use and distribution in any medium, provided the original work is properly cited, the use is non-commercial and no modifications or adaptations are made.

In the United Kingdom, donation after circulatory death (DCD) kidney transplant activity has increased rapidly, but marked regional variation persists. We report how increased DCD kidney transplant activity influenced waitlisted outcomes for a single center. Between 2002-2003 and 2011-2012, 430 (54\%) DCD and $361(46 \%)$ donation after brain death (DBD) kidney-only transplants were performed at the Cambridge Transplant Centre, with a higher proportion of DCD donors fulfilling expanded criteria status (41\% DCD vs. $32 \%$ DBD; $p=0.01$ ). Compared with U.K. outcomes, for which the proportion of DCD: DBD kidney transplants performed is lower $125 \%$; $p<0.0001)$, listed patients at our center waited less time for transplantation (645 vs. 1045 days; p < 0.0001), and our center had higher transplantation rates and lower numbers of waiting list deaths. This was most apparent for older patients laged $>65$ years; waiting time 730 vs. 1357 days nationally; p $<0.001$ ), who received predominantly DCD kidneys from older donors (mean donor age 64 years), whereas younger recipients received equal proportions of living donor, DBD and DCD kidney transplants. Death-censored kidney graft survival was nevertheless comparable for younger and older recipients, although transplantation conferred a survival benefit from listing for only younger recipients. Local expansion in DCD kidney transplant activity improves survival outcomes for younger patients and addresses inequity of access to transplantation for older recipients.

Abbreviations: $\mathrm{Cl}$, confidence interval; CTC, Cambridge Transplant Centre; DBD, donation after brain death; DCD, donation after circulatory death; ECD, expanded criteria donor; ESRF, end-stage renal failure; IQR, interquartile range; KAS, kidney allocation score; KDPI, Kidney Donor Profile Index; LD, living donor; NHSBT, National Health Service Blood Transplant; SCD, standard criteria donor

Received 05 January 2016, revised 24 June 2016 and accepted for publication 12 July 2016

\section{Introduction}

The number of kidney transplants from donation after circulatory death (DCD) donors has risen rapidly in the United Kingdom over the past 10 years (1). From a baseline of $\approx 100$ transplants per annum prior to 2003-2004, >800 DCD kidney transplants were performed in 2013-2014, with DCD transplantation now representing $\approx 40 \%$ of all deceased donor renal transplant activity in the United Kingdom. Worldwide, only the Netherlands achieves similar DCD donor rates per million population (1).

Several aspects of this expansion merit consideration. First, expansion has been accomplished through acceptance of increasingly older donors. In 2003-2004, the mean age of DCD kidney donors nationally was 43 years, whereas in 2013-2014, the mean age was 54 years, significantly higher than the contemporaneous donation after brain death (DBD) cohort (mean age 49 years, $p<0.0001$ ). Consequently, almost half of the DCD kidneys currently transplanted in the United Kingdom come from expanded criteria donors (ECDs) on the basis of donor age $>60$ years. Second, the expansion of DCD kidney transplantation in the United Kingdom has not occurred uniformly, and marked regional differences persist in DCD donation rates (2). This is important because, 
unlike DBD kidneys, DCD kidneys were not allocated nationally until September 2014 (3); instead, both kidneys were offered to the local renal transplant center. Consequently, DCD kidney transplant activity in the different U.K. renal transplant centers varies widely, representing $<10 \%$ of deceased donor transplant activity at some centers and $>60 \%$ at others (4).

Following the introduction of a program to expand DCD kidney transplantation (5-7), the Cambridge Transplant Centre (CTC) now performs approximately twice as many DCD as DBD kidney transplants. Studies to date have generally focused on comparing outcomes of DCD and DBD kidneys from transplantation (5-14); however, given the difference in allocation policies for DCD and DBD kidneys, for which use of a local DCD resource will affect access to the national DBD pool, we sought to examine how alteration in the balance of deceased kidney transplants affected outcomes from listing, with particular consideration of whether any distinct waiting list cohort had been more or less advantaged. Because variation in patterns of DCD kidney transplantation across U.K. transplant centers persists, this further enabled the analysis of outcomes from listing for CTC patients to be compared with those achieved by units in which DCD kidney transplant activity had not increased as dramatically. In the absence of a prospective trial, such comparison provides a means of validating that observed differences were related to different DCD kidney transplant practices.

\section{Materials and Methods}

\section{Study population and design}

The study population comprised all adult patients laged $\geq 16$ years, $n=1459$ ) listed for deceased renal transplantation at our center from August 1, 2002, to July 31, 2012. Recipients were categorized according to age at listing $(<45,45-65$ and $\geq 65$ years) with the following exclusion criteria: multiple organ transplantation $(n=36)$, transfer to our center while on the waiting list $(n=10)$, transfer away from our center while on the waiting list $(n=22)$ and travel overseas for transplantation $(n=10)$. During this period, another eight patients received living donor kidney transplants but were never listed for deceased donor transplantation and thus were not included in the analysis. National data (with data for the CTC omitted) were obtained from the U.K. transplant registry database held by National Health Service Blood and Transplant (NHSBT) only, whereas data for the CTC were amalgamated from case notes, a prospectively maintained local database and the NHSBT database.

Study end points were defined as patient death, end of engagement with the local center (including transferring out of area) or formal study completion (July 31, 2012), with the primary outcome being the fate of the listed patients: progression to transplantation, removal from the waiting list without transplantation, suspension from the waiting list (generally because of illness from which recovery was anticipated), continuation of active listing and death. Patients listed or transplanted from 2009 onward were assumed to have correct follow-up entered in the local and NHSBT databases; cases prior to 2009 were reviewed individually to identify follow-up dates. All-cause graft survival was defined as the time of transplant to the time of return to renal replacement therapy or death. Patient survival was defined as the time from listing to death. For the purposes of outcome from listing, patients who were relisted after a previously failed transplant, even if previously included, were considered as new listings $(n=207)$. To demonstrate how grafts from deceased donors used at the CTC compared with those used elsewhere in the United Kingdom, data were obtained from NHSBT for all grafts from deceased donors transplanted into adult recipients (aged $>18$ years) at the CTC and in the rest of the United Kingdom between 2002 and 2012. Donor age, type (DCD vs. DBD) and criteria (standard vs. expanded) were analyzed according to financial year (April 1 to March 31).

All DCD kidneys were procured from controlled Maastricht category 3/4 (15) donors who incurred irrecoverable brain injury but did not meet the criteria for diagnosis of brain stem death. DCD organ procurement for our center has been detailed previously $(5-7,16,17)$ and differs from standard U.S. practice in that organ donation was pursued for a minimum of $4 \mathrm{~h}$ after withdrawal of life-supporting treatment, regardless of the agonal phase characteristics (6). ECDs were defined as those aged $\geq 60$ years or 50-59 years with two of the following three features: hypertension, terminal serum creatinine $>132 \mathrm{mmol} / \mathrm{L}$ or death from cerebrovascular accident (18). Donors with acute kidney injury (high terminal creatinine) were considered for kidney donation only when recent tests indicated satisfactory baseline renal function; 49 patients received kidneys from donors with terminal creatinine $>200 \mathrm{mmol} / \mathrm{L}$ (range $200-504 \mathrm{mmol} / \mathrm{L}$ ).

\section{Organ allocation}

Kidneys from DBD donors were allocated according to the national algorithm as outlined by NHSBT (the Deceased Donor Kidney Allocation Scheme (3)): Pediatric patients were favored over adult patients, and then 000 HLA-mismatched kidneys were prioritized (with preference to highly sensitized or HLA-homozygous recipients). Beyond this, allocation was based on a point system according to an algorithm incorporating waiting time, HLA match, age, age difference, geographic location, blood group and HLA type. Although the allocation algorithm was altered in 2006, the same algorithm applied to all patients on the waiting list at any one time, and there was no difference in the age-related components of the algorithms. During the study period, DCD kidneys were not allocated nationally, but DCD kidneys were offered on a regional basis (20 regions in the United Kingdom) to the local transplant center that covered that region. The centers were at liberty to select recipients who were considered most suitable for these kidneys, and no formal arrangement existed for sharing with other regional centers. At the CTC, an algorithm similar to that used nationally for DBD kidneys was used to select recipients for DCD kidneys but with the ability to limit the algorithm to select only older recipients (typically aged $>60$ years) for kidneys deemed more "marginal" at the discretion of the on-call team. As described previously $(5,7)$, preimplantation biopsy analysis was used routinely to help determine the suitability of older deceased donor kidneys for transplantation. Living donor transplants were arranged according to standard U.K. practice.

\section{Data and statistical analysis}

Data obtained for each patient included date of birth, sex, date of endstage renal failure (ESRF) diagnosis, date of preemptive listing, date of graft failure if previously transplanted, cause of ESRF, previous transplantation, date of listing, date of transplantation, date of death, cause of death, date of removal from list, date of suspension from list, date of graft loss, cause of graft loss, donor age and donor type (DBD, DCD or living). Graft and patient survival were calculated using Kaplan-Meier estimates and log-rank tests for statistical significance. In the comparison of patient survival from listing (Figure $6 \mathrm{C}$ ), only survival from the time of listing for the first transplant was analyzed. For the older cohort, comparison of survival for those transplanted against those remaining on the waiting list was also assessed by the time-dependent nonproportional 


\section{Waitlisted Outcomes for DCD Renal Transplantation}

Cox regression model (19), adjusting for primary disease, ethnicity, sex, blood group, BMI, and sensitization status. Median waiting times for deceased donor kidney transplantation were calculated using the KaplanMeier method of estimation excluding patients who were transplanted using a kidney from a living donor and censoring for removal (ill health or recovery of renal function) or deaths on the list. Rates of delisting for transplantation (death or removal from the list without transplantation) were also estimated by Kaplan-Meier analysis censoring for transplantation. The Kidney Donor Profile Index (KDPI) was calculated using the 2014 reference data set (20). Categorical data were examined with the chi-square test, nonparametric continuous data were assessed using the Kruskal-Wallis test and parametric continuous data were assessed with the Student t-test. Statistical analysis was performed using SAS (v9.1; SAS Institute, Cary, NC) and GraphPad Prism (v5.03; GraphPad Software, La Jolla, CA).

\section{Results}

\section{Changing profile of deceased kidney transplants}

A single-center retrospective observational cohort study was performed to examine outcomes for all adult patients listed for kidney transplantation at the CTC between 2002-2003 and 2011-2012. As reference, the number of DCD adult kidney-only transplants performed increased markedly during this period, from 11 in the 2002-2003 financial year to 68 in the 2011-2012 financial year (Figure 1A); this represents a much higher proportion of deceased donor kidney transplantation than occurred nationally (Figure 1B). Figure 1A also shows that numbers of living donor kidney transplants increased over the study period but to a lesser extent, whereas numbers of DBD kidney transplants remained relatively static. In keeping with national trends, the age of the DCD kidney donors increased throughout the study period (Figure 1C). Consequently, compared with the DBD kidney transplants, a significantly higher proportion of the DCD kidney transplants performed were from ECDs (DBD 33\% [114 of 346] vs. DCD 44\% [186 of 426]; chisquare, $p=0.003$ ).

\section{Older listed patients are less likely to receive a kidney transplant}

To assess how the expansion in availability of predominantly older DCD kidneys affected the recipient pool, outcomes from listing for study patients were compared according to three groups of listed patients: those aged $<45,45-65$ and $>65$ years at listing for kidney transplantation (Table 1). Transplant outcomes and donor details are listed in Table 2. Over the entire study period, older listed patients were less likely to be transplanted and more likely to be removed from the waiting list than younger patients (Table 2). Nevertheless, comparison of outcomes from listing at 1, 3 and 5 years with national data (Figure 2) demonstrated for all age groups that patients on the CTC waiting list were more likely to be transplanted than their equivalent U.K. cohorts. This difference was more marked for the older group: whereas $59 \%$ of CTC-listed patients aged >65 years were transplanted by 5 years, only $38 \%$ of the equivalent national waiting pool had received a kidney transplant (chi-square, $p<0.001$ ). Conversely, a greater proportion of older listed patients nationally were either removed from the list or died before transplantation (at 5 years: CTC $41 \%$ vs. national 46\%; $p<0.001$ ).

\section{Higher rates of delisting for older patients on the waiting list}

Comparison of the rates of removal of patients from the waiting list because of death or ill health was further assessed by Kaplan-Meier analysis of outcomes for the Cambridge Transplant Centre-listed patients (Figure 3). This analysis confirmed that rates of delisting were significantly higher for the cohort of older listed patients, with $<50 \%$ of patients aged $>65$ years who were listed but not transplanted still active on the list after 4 years. Analysis of the U.K. data set also revealed that the rate of removal from the waiting list was significantly higher for the older cohort but that for each age range, delisting rates were similar to those observed at the CTC (Figure 3), suggesting that the smaller proportion of older patients dying or being removed from the waiting list at our center does not reflect a bias toward listing relatively more fit recipients.

\section{Donor profiles differ markedly according to recipient age}

In considering why older listed patients at the CTC have greater access to transplantation, analysis of the types of kidneys transplanted into each recipient age group revealed marked differences (Table 2). Whereas the proportions of living donor, DCD and DBD kidney transplants were broadly equivalent for listed recipients aged $<45$ years, patients aged $>65$ years received kidney transplants from predominantly DCD donors, with DBD and living donors together constituting only approximately one-third of the transplants performed. Numerically, most DCD kidneys were nevertheless transplanted into recipients in the younger age groups (Table 2 ).

In keeping with the older age of DCD kidney donors, older patients generally received kidney transplants from older donors (Figure 4, Table 2). Calculation of the KDPI donor score revealed that these kidneys could be considered extremely marginal (Table 2), with 69\% of kidneys transplanted into the older cohort scoring $>85$.

The relatively rapid rate of removal of older patients from the waiting list due to death or illness suggests that local availability of DCD kidneys may be a particularly important factor underlying the difference in CTC and national transplantation rates for older recipients. In support, comparison of CTC and national waiting times for kidney transplantation (Figure 5) highlights that waiting times for the three recipient age groups were comparable for CTC patients but that older patients waited substantially longer nationally. Moreover, waiting times for our older 
A

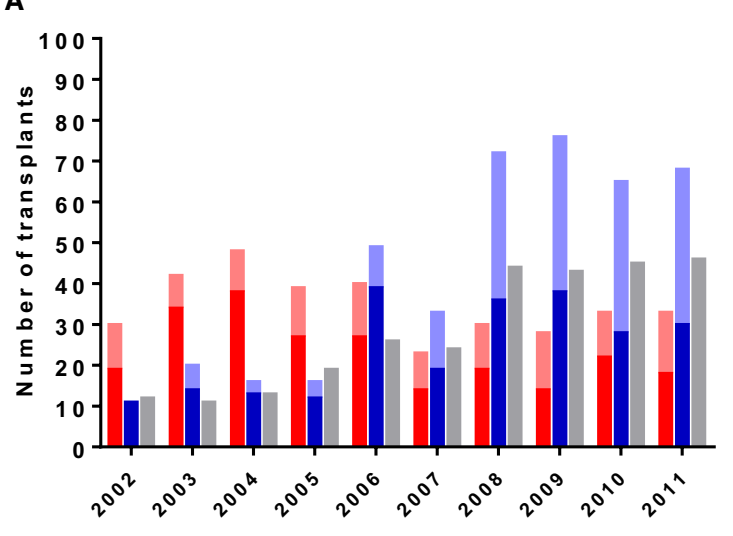

B

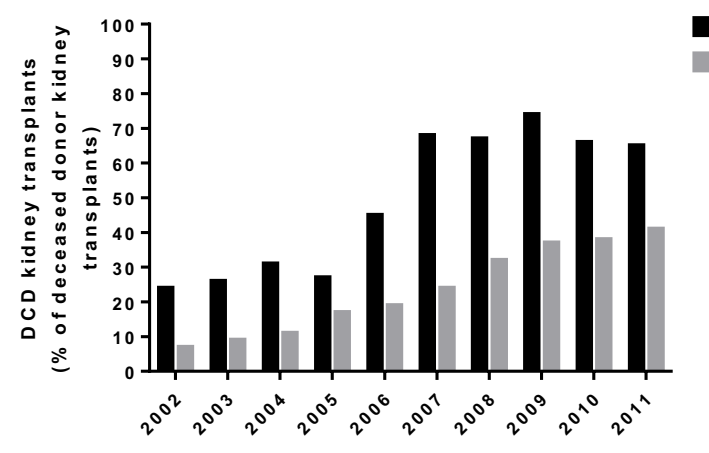

C

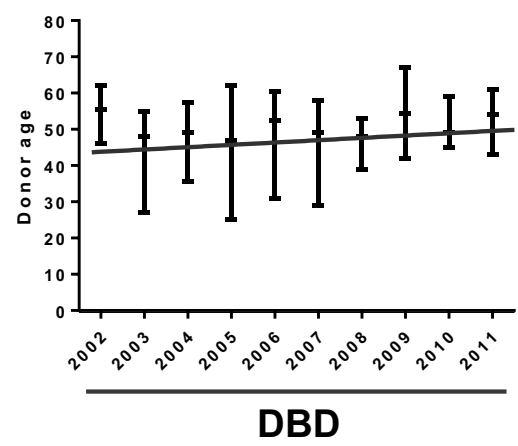

Figure 1: Changing profiles of deceased kidney donors. (A) Numbers of expanded criteria donor (ECD) and standard criteria donor (SCD) kidneys transplanted at the Cambridge Transplant Centre (CTC) according to deceased donor type (donation after brain death [DBD] and donation after circulatory death [DCD]). Numbers of living donor (LD) transplants are included for comparison. (B) DCD kidney transplants, as a percentage of deceased donor kidney transplants at the CTC and in the United Kingdom. (C) Regression lines of donor age superimposed on graphs depicting median and interquartile range of donor age per year for CTC DBD and DCD donors: $p=0.047$, analysis of covariance. Year denotes financial year.

patients fell during the study period (Figure 5); presumably reflecting the continued expansion of the DCD kidney transplant program. Although the simultaneous increase in living donor transplant activity at our center (Figure 1A) would be expected to contribute to a fall in waiting times, the impact is likely to be less pronounced than for the DCD kidney transplant program because substantially fewer living donor kidney transplants were performed at our center during the study period than DCD kidney transplants (283 vs. 426 including eight living donor recipients never listed for a deceased donor transplant) (Figure 1A). It should also be noted that compared with the United Kingdom, our living donor program remained relatively small during 
Waitlisted Outcomes for DCD Renal Transplantation

Table 1: Baseline demographics of patients listed for renal transplantation at the Cambridge Transplant Centre between $1^{\text {st }}$ August 2002 and $31^{\text {st }}$ July 2012

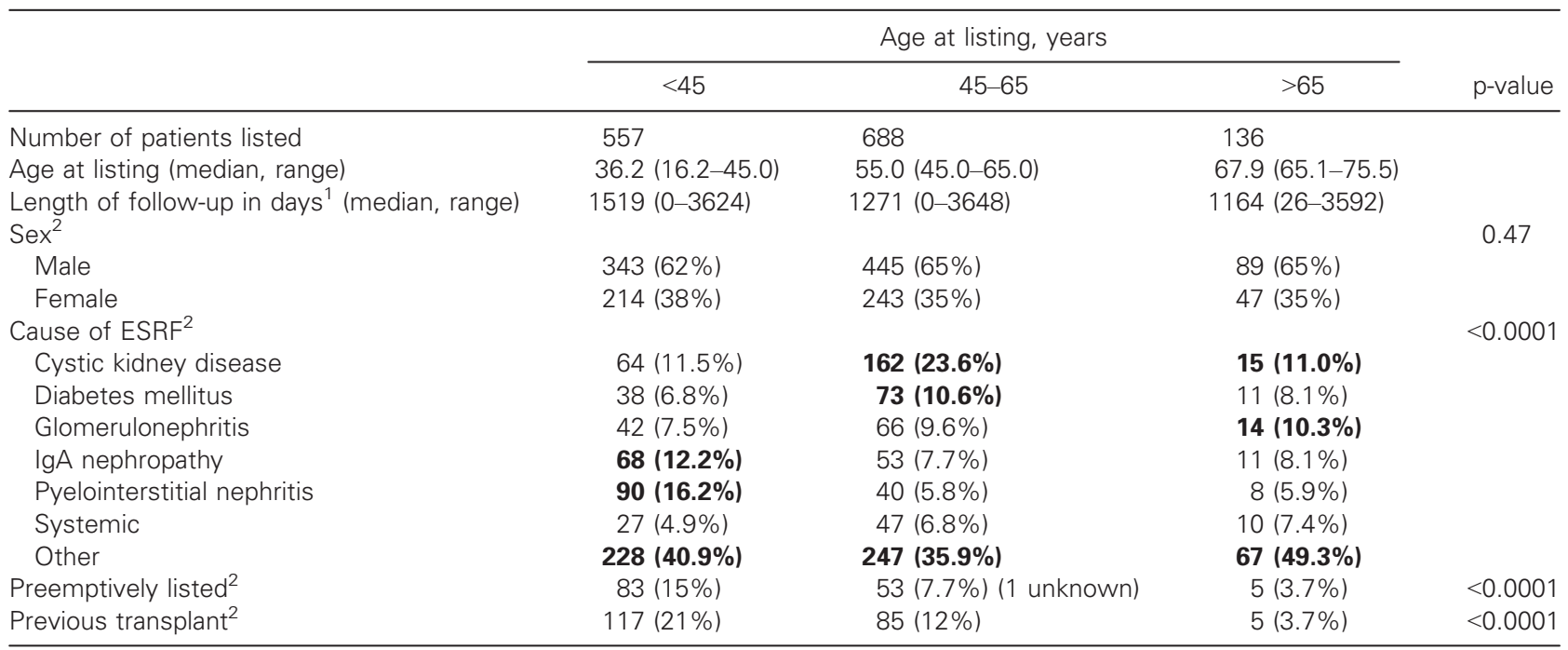

ESRF, end-stage renal failure. Values in bold indicate three highest causes of ESRF per age group.

${ }^{1}$ Length of follow-up was from date of listing until patient death, loss of engagement with local center, movement out of region, or end of study period (July 31, 2012).

${ }^{2}$ Chi-squared test.

Table 2: Outcomes on $31^{\text {st }}$ July 2012 of patients listed for renal transplantation at the Cambridge Transplant Centre between $1^{\text {st }}$ August 2002 and $31^{\text {st }}$ July 2012

\begin{tabular}{|c|c|c|c|c|}
\hline & \multicolumn{3}{|c|}{ Age at listing, years } & \multirow[b]{2}{*}{$p$-value } \\
\hline & $<45$ & $45-65$ & $>65$ & \\
\hline Number listed & 557 & 688 & 136 & \\
\hline Outcomes $^{1}$ & & & & $<0.0001$ \\
\hline Transplanted & $411(73.8 \%)$ & $479(69.6 \%)$ & 72 (52.9\%) & \\
\hline Suspended & $42(7.5 \%)$ & $115(16.7 \%)$ & $9(6.6 \%)$ & \\
\hline Removed or died & $20(3.6 \%)$ & $14(2.0 \%)$ & $30(22.1 \%)$ & \\
\hline Remained active & $84(15.1 \%)$ & $80(11.6 \%)$ & $25(18.4 \%)$ & \\
\hline Age at transplantation (median, range) & $37(17-49)$ & $57(45-70)$ & $69(66-75)$ & \\
\hline Donor age ${ }^{2}$ (median, range) & $44(1-70)$ & $56(2-82)$ & $67(23-79)$ & $<0.0001$ \\
\hline Donor type $(\%)^{1}$ & & & & $<0.0001$ \\
\hline DBD & $127(31 \%)$ & $119(25 \%)$ & $17(24 \%)$ & \\
\hline DCD & $130(32 \%)$ & $243(51 \%)$ & $45(63 \%)$ & \\
\hline Live donor & $154(37 \%)$ & $117(24 \%)$ & $10(14 \%)$ & \\
\hline $\mathrm{KDPI}^{2,3}$ (median, range) & $46(1-100)$ & $77(1-100)$ & $94(18-100)$ & $<0.0001$ \\
\hline \multicolumn{5}{|l|}{ Graft failure (\% of transplanted) } \\
\hline All cause & $49(11.9 \%)$ & $60(12.5 \%)$ & $18(25 \%)$ & \\
\hline Censored for death & $42(10.2 \%)$ & $45(9.4 \%)$ & $6(8.3 \%)$ & \\
\hline Death (\% of listed) & $40(7.2 \%)$ & $59(8.6 \%)$ & $26(19 \%)$ & \\
\hline With transplant & $13(2.3 \%)$ & $20(2.9 \%)$ & $15(11 \%)$ & \\
\hline Without transplant & $27(4.8 \%)$ & $39(5.7 \%)$ & $11(8.1 \%)$ & \\
\hline
\end{tabular}

DBD, donation after brain death; DCD, donation after circulatory death; KDPI, Kidney Donor Profile Index.

${ }^{1}$ Chi-squared analysis.

${ }^{2}$ Kruskal-Wallis analysis.

${ }^{3}$ Kidney donor profile index was calculated for deceased donors only using the 2014 dataset.

the study period (283 of 1088 [26\%] kidney transplants performed at the CTC vs. 7976 of 22163 [36\%] performed nationally; $p<0.0001)$. Similarly, the fall in CTC waiting times was most apparent for the recipient groups aged 45-65 and >65 years (Figure 5), and in both cohorts, DCD transplant activity was dominant (Table 2).

The reason that older U.K. patients are apparently disadvantaged in their access to DBD kidney transplantation is 

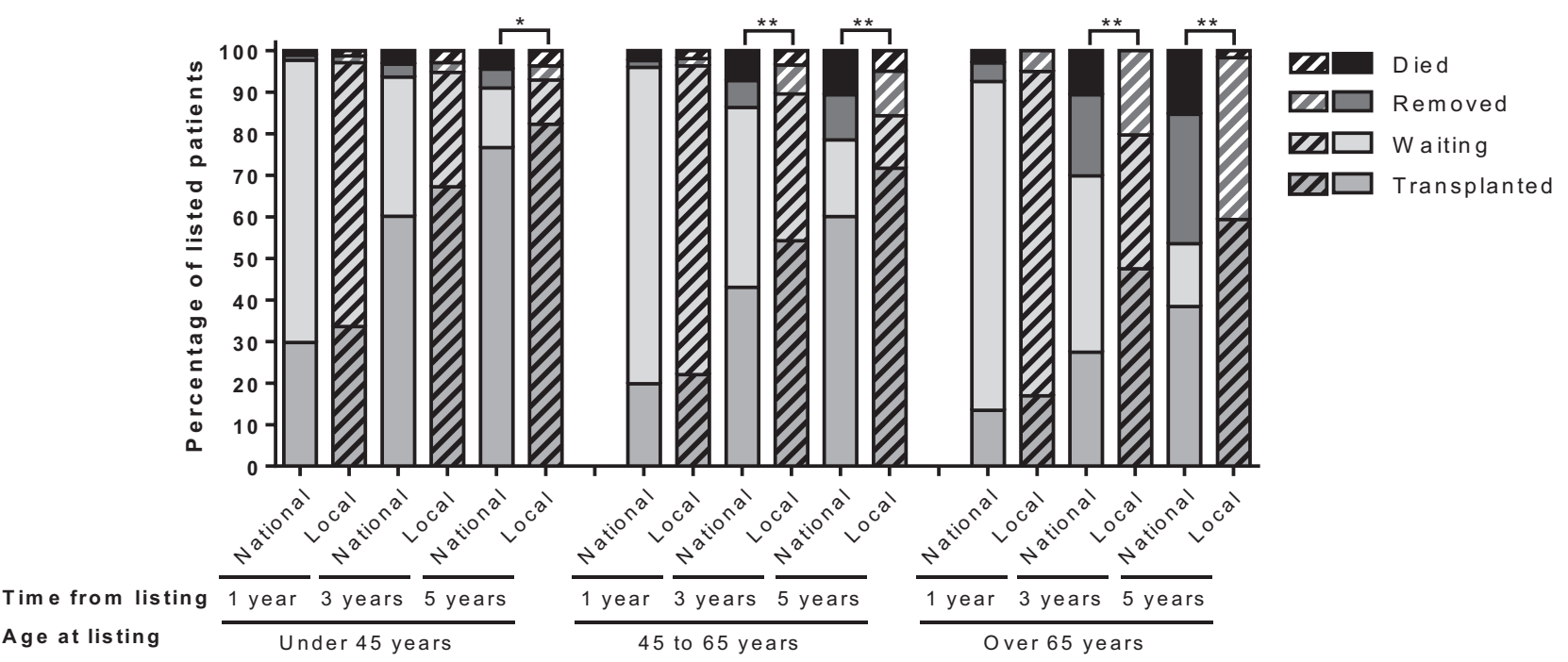

Figure 2: Comparison of outcomes for waitlisted patients nationally and at the Cambridge Transplant Centre (CTC). Waiting list status at 1, 3, and 5 years from listing of patients listed for renal transplantation between 2002 and 2008 nationally and locally (at the (TC) according to age at listing: 7118,8620 , and 1837 patients aged $<45,45-65$, and $>65$ years, respectively, were listed nationally, and 375,459 , and 59 local patients were listed, respectively, for the same age groups. ${ }^{*} p<0.05$; ${ }^{*} p<0.001$; Fisher exact test.

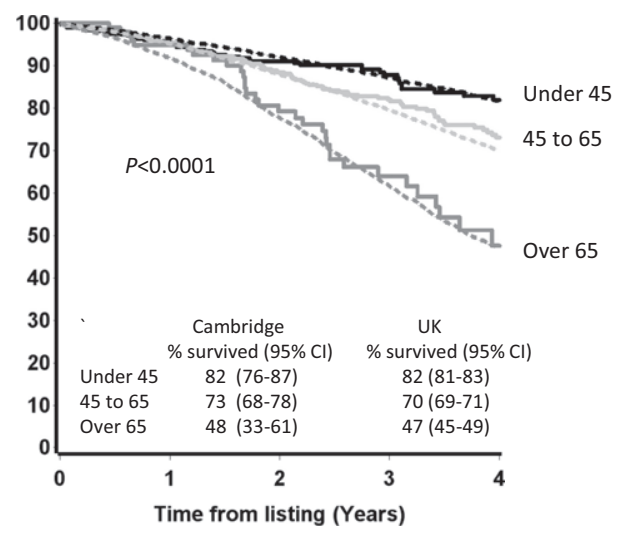

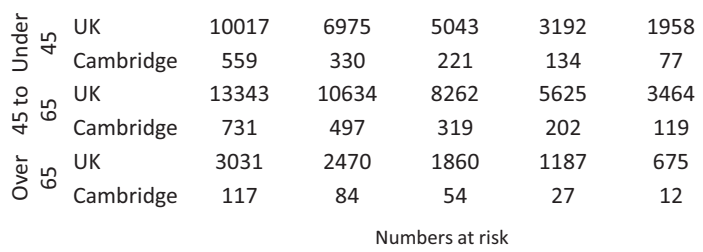

Figure 3: Rates of delisting from kidney transplant waiting list. Kaplan-Meier analysis of rates of delisting (due to death or removal from the waiting list and censored for transplantation) for patients listed at the Cambridge Transplant Centre (CTC; dotted line) and in the rest of the United Kingdom (solid line), according to age at listing for transplantation. Log-rank analysis confirms that at the CTC, older patients (aged $>65$ years) were delisted significantly more rapidly than younger patients $(p<0.01)$ but that for any age cohort, local (CTC) rates of delisting were not different from U.K. rates ( $p$-value not significant). $\mathrm{Cl}$, confidence interval.

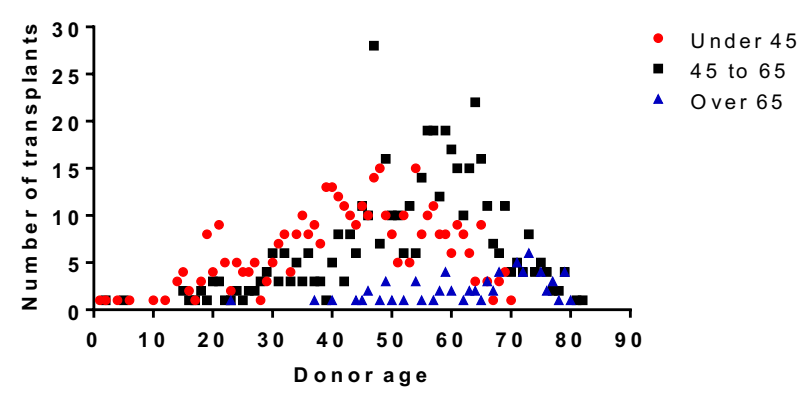

Figure 4: Differences in donor profiles according to recipient age at listing. Scatter plot depicting deceased donor age for each recipient group (Kruskal-Wallis, $p<0.0001$ ).

not immediately obvious, although we thought it likely to be a facet of the algorithm for national sharing of DBD kidneys (21), whereby younger recipients of DBD kidneys generally score more highly than older recipients. Consequently, older recipients must wait longer to accrue sufficient waiting time points through the algorithm to outcompete younger recipients (Figure 5, Table 3). Although DCD kidneys were not allocated nationally during the study period, application of the algorithm to our recipients revealed that for all age groups, the allocation score was less for CTC DCD recipients than for nationally allocated DBD kidneys (Table 3). This is partly due to the smaller local recipient pool that generally prevents close HLA matching between donor and recipient but mostly reflects the much shorter waiting times for transplantation. 


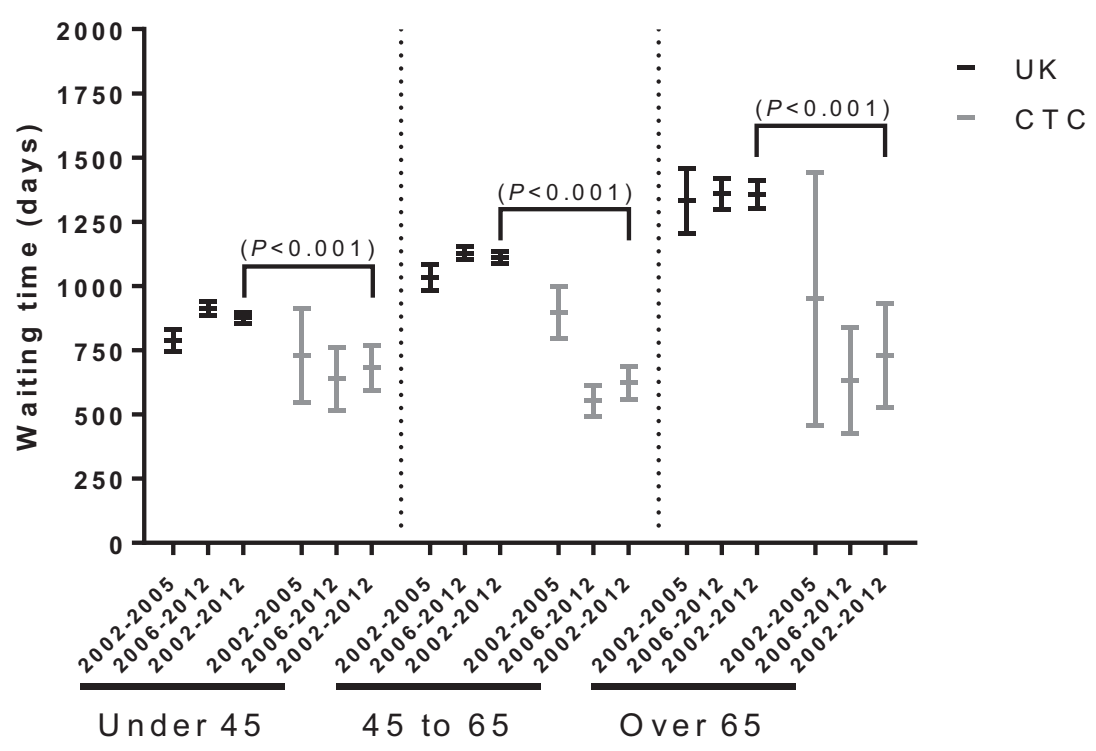

Figure 5: National and Cambridge Transplant Centre (CTC) waiting times according to recipient age. Waiting time to adult kidney transplantation in the United Kingdom and at the CTC according to age at listing and year of listing. Graph shows median \pm confidence intervals, and p-values depict log-rank analysis.

Table 3: Kidney Allocation Scores (KAS) for deceased donor transplants from 3 April 2006 to 31 December 2012 for national DBD kidneys and from 3 April 2006 to 31 July 2012 for local DCD kidneys

\begin{tabular}{lcclccc}
\hline & \multicolumn{2}{c}{ National DBD kidney transplants } & & \multicolumn{2}{c}{ Local DCD kidney transplants } \\
\cline { 2 - 3 } Age at listing, years & Number of transplants & KAS (median IQR) & & Number of transplants & KAS (median, IQR) & p-value \\
\hline$<45$ & 2593 & $3201(2709-3757)$ & & 107 & $2178(1563-2609)$ & $<0.0001$ \\
$45-65$ & 3274 & $2771(2156-3332)$ & & 200 & $1582(1300.3-2002)$ & $<0.0001$ \\
$>65$ & 654 & $2327(1622-2909)$ & & 33 & $1385(1044.5-1603)$ & $<0.0001$ \\
\hline
\end{tabular}

$\mathrm{DBD}$, donation after brain death; DCD, donation after circulatory death; IQR, interquartile range.

\section{Kidney transplant outcome and patient survival from listing}

Outcomes for DCD kidney transplantation at our unit were described previously (5-7), and similar outcomes for DCD and DBD kidney transplantation were reported. All-cause kidney graft survival was poorer for older recipients (Figure 6A) but largely reflected that 12 of 18 graft losses in this group were due to recipient death (generally due to malignancy or sepsis) with a functioning graft (median preterminal creatinine $151 \mu \mathrm{mol} / \mathrm{L}$ ). Graft survival when censored for recipient death was comparable for the three recipient groups (Figure 6B). In anticipation that the impact of increased kidney transplant activity would be most apparent in an assessment of recipient outcomes that included time on the waiting list, recipient survival for each age group was assessed from the point of listing and compared with those listed patients who did not receive a transplant (Figure 6C). This analysis confirmed that transplantation was associated with improved survival for patients in the two younger age groups but not for the older cohort. An additional nonproportional Cox regression model analysis (19) was performed to compare survival for the older patients who received a transplant against those who remained on the waiting list. This analysis also failed to show a survival advantage for transplantation in the older group (hazard ratio 1.87 [95\% confidence interval $0.42-8.26$ ]; $p=0.4$ ).

\section{Discussion}

The rate of expansion of DCD kidney transplantation in the United Kingdom has been remarkable; however, marked variations in regional intensive care unit DCD donation rates (2), together with apparent differences among transplant centers in assessment of suitability of DCD kidneys for transplantation, have resulted in geographic fragmentation of current DCD kidney transplant activity. The major consequence of this piecemeal implementation is that transplanting increasing numbers of 
A

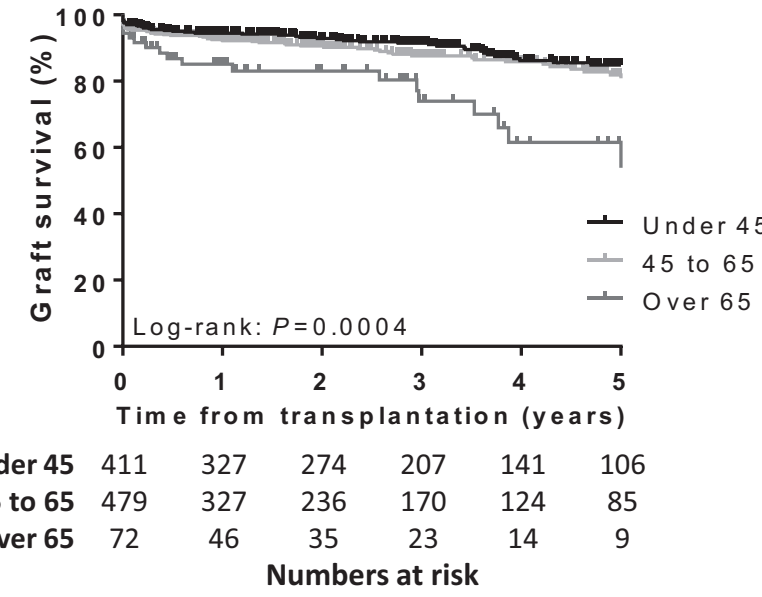

B

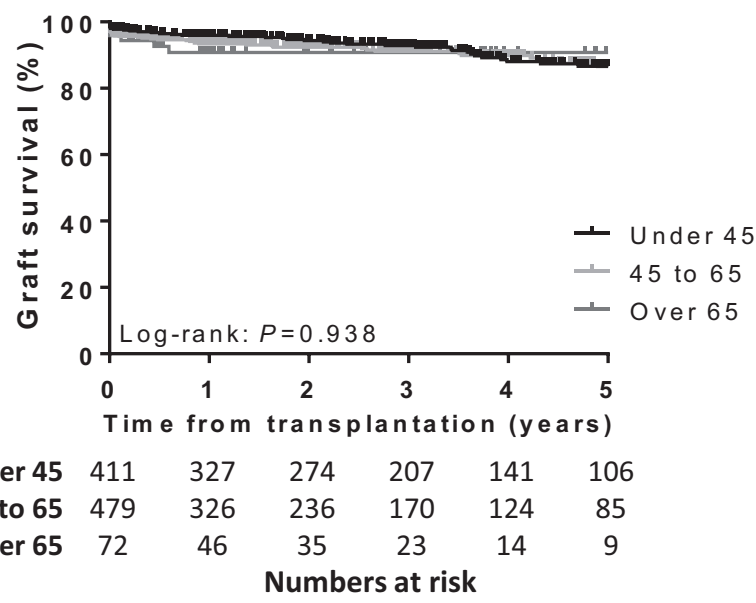

C

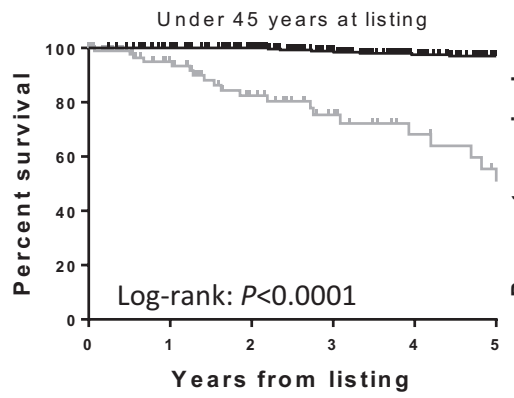

Transplanted $341 \quad 325 \quad 289 \quad 248 \quad 201 \quad 163$ $\begin{array}{lllllll}\text { Not transplanted } & 99 & 62 & 42 & 27 & 18 & 13\end{array}$
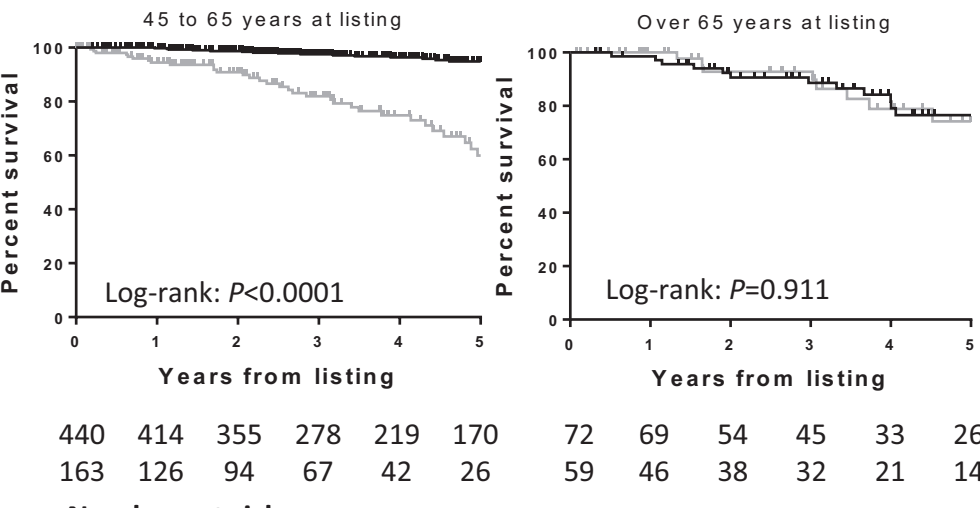

Numbers at risk

Figure 6: Kidney graft and patient survival. Kaplan-Meier curves and numbers at risk for (A) all-cause graft survival ( $p=0.0004$; log-rank test) and (B) graft survival censored for death as a cause of graft loss according to age at listing ( $p=0.2705)$. (C) KaplanMeier curves and numbers at risk for patient survival from first listing in transplanted (black line) and nontransplanted (gray line) patients for those aged $<45$ years (left), 45-65 years (middle) and $>65$ years (right) at time of listing.

locally resourced DCD kidneys will, for a particular center, likely restrict access of that center's listed population to the nationally allocated DBD kidney pool; a major factor determining national allocation is waiting time for a transplant. The impact of this apparent conflict between local DCD kidney and national DBD kidney allocation would be potentially more marked if, as was once generally considered, DCD kidneys were of inherently poorer quality than DBD kidneys; however, analyses of individual-center $(5,7)$ and national U.K. transplant data $(8,9)$ have demonstrated that age-matched outcomes for DCD and DBD kidney transplantation are similar. Nevertheless, increased use of a local DCD donor pool would potentially result in transplantation of less well-matched kidneys from older donors than the recipients would have received otherwise from the national DBD pool. Consequently, we considered it important to analyze how the marked expansion in DCD kidney transplantation at our center affected recipient outcomes but to focus this analysis on outcomes from listing for various age groups of the waiting list population. Our analysis suggested that waiting times for transplantation are improved for all recipients and that, given the equivalent outcomes from transplantation for DCD and DBD kidneys in our center (5-7), this is associated with improved survival outcomes from listing of recipients aged $<65$ years. The situation is more complex for older recipients because, although this group arguably gains most in terms of equity of access to transplantation, their survival is not enhanced by transplantation.

There are potentially several reasons why older listed patients are generally disadvantaged in their access to transplantation. Our analysis highlights that options for living donor transplantation for our older recipients are generally more limited than for younger recipients, 
Waitlisted Outcomes for DCD Renal Transplantation

presumably either because of concerns relating to fitness of the spouse or because living donation from offspring to parent is not perceived as being as acceptable as parent-to-offspring donation. The apparent inequity of access of older recipients to the national DBD pool is largely due to the U.K. matching algorithm for DBD kidney allocation (21). The algorithm calculates an allocation score for level 1, 2, and 3 HLA-mismatched kidneys based on, among other factors, waiting-time for transplantation, the degree of HLA matching and age difference between donor and recipient, but it was designed specifically "to ensure well-matched transplants for younger patients while recognizing that $\mathrm{HLA}$ matching is less important for older patients." Consequently, the algorithm is weighted to preferentially allocate more closely HLA-matched kidneys to younger recipients, regardless of donor-recipient age difference. Accordingly, younger recipients receive an exponentially greater number of points than older recipients for receipt of a well-matched kidney, and a crude analysis of the algorithm suggests that the older cohort would need to accrue $\approx 4$ years of waiting-time points to compensate for this bias toward younger recipients. This carries the major drawback that, as highlighted by our analysis, the window for transplantation of older listed recipients is small and that approximately half of these patients will either have died or become too frail to transplant after 4 years. This presumably explains why, when compared with younger listed recipients, only approximately half as many older listed patients nationally receive a transplant and yet, at 5 years, the percentage of patients still waiting for transplantation is similar for both groups $(\approx 15 \%)$ (Figure 2).

We ostensibly adopted the same algorithm used nationally for allocating local DCD kidneys to our recipients, and it is perhaps surprising that this enabled us to transplant our older recipients. Our older recipients generally received kidneys from older DCD donors (Table 2), and we think this is partly a consequence of a relative reduction, compared with the national DBD pool, in the size of the local DCD donor pool that limits availability of wellmatched younger recipients for a proportion of the older DCD donors. Nevertheless, local allocation of DCD kidneys provided the flexibility to impose additional restrictions on recipient selection. We have also, at the clinicians' discretion, deliberately chosen to allocate kidneys from older DCD donors that were deemed more marginal to only older recipients, and it is notable that a substantially higher proportion of the DCD than DBD kidneys transplanted fulfilled ECD status. This mirrors the strategy adopted by the Eurotransplant Senior Program (22), in which kidneys from older donors are preferentially transplanted into older recipients. Our analysis provides further support for the benefits of this approach. The kidneys transplanted into our older recipients (mainly from older DCD donors) could be considered extremely marginal, with $69 \%$ having a KDPI score $>85$, and yet graft outcomes were generally favorable and prejudiced mainly by recipient death with a functioning graft. The surprisingly good outcomes for these kidneys may reflect our routine use of preimplantation biopsy analysis to help determine suitability for transplantation $(5,7)$.

It is clear that the availability of locally allocated DCD kidneys has enabled us to transplant a far greater proportion of our transplant waiting list than would otherwise be the case. The short waiting time for transplantation achieved, currently the lowest in the United Kingdom (23), has substantially improved the opportunity for transplanting our older listed recipients. As the hemodialysis population ages (24), it is likely that greater numbers of older patients will continue to be listed for kidney transplantation; their deliberate selection as recipients of older DCD kidneys may provide an opportunity for transplantation not otherwise afforded. In this regard, our findings are likely to have relevance beyond U.K. transplant practice. Death on the waiting list appears to be an even greater problem for older patients in the United States (25), and yet the recent introduction of the U.S. Kidney Allocation System has been associated with increased discard rates of kidneys with the highest KDPI scores (26) and has seen a decrease in transplant rates for older listed patients and an increase in their waiting time for transplant (27). Given that kidneys with high KDPI are typically from DCD ECDs and that such kidneys are allocated locally rather than nationally, our findings suggest that their preferential allocation to older listed patients may provide an opportunity for transplantation for these recipients that would not otherwise be available.

A concern raised by our analysis is whether it is appropriate, in the absence of a demonstrable survival benefit for transplantation, to continue to allocate organs to older recipients that may otherwise improve the survival of younger recipients. The apparent lack of survival benefit for transplantation in our older cohort is surprising because previous analyses from the United Kingdom have suggested otherwise $(28,29)$, but it does not appear to relate to the limited number of older patients in our study. In contrast to the younger cohorts, the survival curves for the older transplanted patients and the older patients that remained on dialysis are closely matched (Figure 6C). This may relate to continuing improvements in survival for older patients with ESRF on hemodialysis (24). Nevertheless, we considered it justifiable to continue to list older recipients on the basis of patient choice and improvement in quality of life $(30,31)$. In addition, even allowing for deliberate selection of older recipients for kidneys deemed more marginal, proportionally fewer older than younger listed patients ultimately received a kidney transplant (Table 2); therefore, it is difficult to argue that older patients have been unfairly advantaged by our selection policy. Moreover, those patients aged $>65$ years listed 
during the study period represented $<10 \%$ of all listed patients, and it should be stressed that the majority (89\%) of DCD kidneys in our study were transplanted into patients aged $<65$ years at time of listing. Consequently, although the CTC DCD program appears to perhaps have made the biggest difference in outcomes for older listed patients, the greatest impact has instead been on younger listed patients, with many more transplants realized by the program conferring improved survival from listing.

In summary, our analysis demonstrated that local expansion of DCD transplant activity can improve outcomes for younger and older listed patients. For older listed recipients, an awareness that their window for transplantation is likely to be narrow may justify increased use of marginal kidneys with high KDPI scores.

\section{Acknowledgments}

The authors acknowledge support from the National Institute of Health Research Cambridge Biomedical Research Centre and the NIHR Blood and Transplant Research Unit in Organ Donation and Transplantation at the University of Cambridge in collaboration with Newcastle University and in partnership with NHS Blood and Transplant (NHSBT). B.M.-S. is funded by a National Institute of Health Research Academic Clinical Fellowship. The authors would like to thank Mark Thomas and Adam Venn for assistance with data collection from the local transplant database.

\section{Disclaimer}

The views expressed in this manuscript are those of the authors and not necessarily those of the NHS, the NIHR, the Department of Health or NHSBT National Institute of Health Research Blood and Transplant Research Unit.

\section{Disclosure}

The authors of this manuscript have no conflicts of interest to disclose as described by the American Journal of Transplantation.

\section{References}

1. Summers DM, Watson CJ, Pettigrew GJ, et al. Kidney donation after circulatory death (DCD): State of the art. Kidney Int 2015; 88: 241-249.

2. Summers DM, Johnson RJ, Hudson AJ, et al. Standardized deceased donor kidney donation rates in the UK reveal marked regional variation and highlight the potential for increasing kidney donation: A prospective cohort studydagger. Br J Anaesth 2014; 113: 83-90.

3. NHSBT. Kidney transplantation: Organ allocation. 2014 [cited last accessed 31st December 2015]. Available from: http:// www.odt.nhs.uk/pdf/kidney_allocation_policy.pdf.
4. NHSBT. Organ donation and transplantation. Activity Report 2014/15. 2015 [cited Last accessed 20th June 2016]. Available from: http://nhsbtmediaservices.blob.core.windows.net/organdonation-assets/pdfs/activity_report_2014_15.pdf.

5. Kosmoliaptsis V, Salji M, Bardsley V, et al. Baseline donor chronic renal injury confers the same transplant survival disadvantage for DCD and DBD kidneys. Am J Transplant 2015; 15: 754-763.

6. Reid AW, Harper S, Jackson $\mathrm{CH}$, et al. Expansion of the kidney donor pool by using cardiac death donors with prolonged time to cardiorespiratory arrest. Am J Transplant 2011; 11: 995-1005.

7. Mallon DH, Riddiough GE, Summers DM, et al. Successful transplantation of kidneys from elderly circulatory death donors by using microscopic and macroscopic characteristics to guide single or dual implantation. Am J Transplant 2015; 15: 2931-2939.

8. Summers DM, Johnson RJ, Allen J, et al. Analysis of factors that affect outcome after transplantation of kidneys donated after cardiac death in the UK: A cohort study. Lancet 2010; 376: 1303-1311.

9. Summers DM, Johnson RJ, Hudson A, Collett D, Watson CJ, Bradley JA. Effect of donor age and cold storage time on outcome in recipients of kidneys donated after circulatory death in the UK: A cohort study. Lancet 2013; 381: 727-734.

10. Singh SK, Kim SJ. Does expanded criteria donor status modify the outcomes of kidney transplantation from donors after cardiac death? Am J Transplant 2013; 13: 329-336.

11. Jochmans I, Moers C, Smits JM, et al. Machine perfusion versus cold storage for the preservation of kidneys donated after cardiac death: A multicenter, randomized, controlled trial. Ann Surg 2010; 252: 756-764.

12. Snoeijs MG, Winkens B, Heemskerk MB, et al. Kidney transplantation from donors after cardiac death: A 25-year experience. Transplantation 2010; 90: 1106-1112.

13. Snoeijs MG, Buurman WA, Christiaans MH, et al. Histological assessment of preimplantation biopsies may improve selection of kidneys from old donors after cardiac death. Am J Transplant 2008; 8: 1844-1851.

14. Tojimbara T, Fuchinoue S, Iwadoh K, et al. Improved outcomes of renal transplantation from cardiac death donors: A 30-year single center experience. Am J Transplant 2007; 7: 609-617.

15. Kootstra G, Daemen JHC, Oomen APA. Categories of nonheart-beating donors. Transpl Proc 1995; 27: 2893-2894.

16. Qureshi MS, Callaghan CJ, Bradley JA, Watson CJ, Pettigrew GJ. Outcomes of simultaneous pancreas-kidney transplantation from brain-dead and controlled circulatory death donors. $\mathrm{Br} \mathrm{J}$ Surg 2012; 99: 831-838.

17. Mallik M, Callaghan CJ, Hope M, et al. Comparison of liver transplantation outcomes from adult split liver and circulatory death donors. Br J Surg 2012; 99: 839-847.

18. Port FK, Bragg-Gresham JL, Metzger RA, et al. Donor characteristics associated with reduced graft survival: An approach to expanding the pool of kidney donors. Transplantation 2002; 74: $1281-1286$.

19. Wolfe RA, Ashby VB, Milford EL, Ojo AO, Ettenger RE, Agodoa LY, et al. Comparison of mortality in all patients on dialysis, patients on dialysis awaiting transplantation, and recipients of a first cadaveric transplant. N Engl J Med 1999; 341: 1725-1730.

20. Israni AK, Salkowski N, Gustafson S, et al. New national allocation policy for deceased donor kidneys in the United States and possible effect on patient outcomes. J Am Soc Nephrol 2014; 25: 1842-1848.

21. Johnson RJ, Fuggle SV, Mumford L, Bradley JA, Forsythe JL, Rudge CJ. A New UK 2006 National Kidney Allocation 


\section{Waitlisted Outcomes for DCD Renal Transplantation}

Scheme for deceased heart-beating donor kidneys. Transplantation 2010; 89: 387-394.

22. Frei U, Noeldeke J, Machold-Fabrizii V, et al. Prospective agematching in elderly kidney transplant recipients-a 5-year analysis of the Eurotransplant Senior Program. Am J Transplant 2008; 8: 50-57.

23. NHSBT. Annual report on kidney transplantation 2014-2015. 2015 [cited last accessed 20th June 2016]. Available from: http:// www.odt.nhs.uk/pdf/organ_specific_report_kidney_2015.pdf.

24. Registry TRAUR. UK Renal Registry 17th Annual Report. 2014 [cited Last accessed 20th June 2016]. Available from: https:// www.renalreg.org/reports/2014-seventeenth-annual-report/.

25. Schold J, Srinivas TR, Sehgal AR, Meier-Kriesche HU. Half of kidney transplant candidates who are older than 60 years now placed on the waiting list will die before receiving a deceaseddonor transplant. Clin J Am Soc Nephrol 2009; 4: 1239-1245.

26. Bae S, Massie AB, Luo X, Anjum S, Desai NM, Segev DL. Changes in discard rate after the introduction of the Kidney
Donor Profile Index (KDPI). Am J Transplant 2016; 16: 2202-2207.

27. Network OPaT. Kidney allocation system (KAS) 'Out-of-the-gate' monitoring report. 2015 [cited Last accessed 20th June 2016]. Available from: http://optn.transplant.hrsa.gov/media/1178/ kas_report_06-2015.pdf.

28. Oniscu GC, Brown H, Forsythe JL. How great is the survival advantage of transplantation over dialysis in elderly patients? Nephrol Dial Transplant 2004; 19: 945-951.

29. Oniscu GC, Brown H, Forsythe JL. Impact of cadaveric renal transplantation on survival in patients listed for transplantation. J Am Soc Nephrol 2005; 16: 1859-1865.

30. Humar A, Denny R, Matas AJ, Najarian JS. Graft and quality of life outcomes in older recipients of a kidney transplant. Exp Clin Transplant 2003; 1: 69-72.

31. Laupacis A, Keown P, Pus N, et al. A study of the quality of life and cost-utility of renal transplantation. Kidney Int 1996; 50: 235-242. 